\title{
REVIEW
}

\section{The pathogenesis of pulmonary fibrosis: a moving target}

\author{
Wim A. Wuyts, Carlo Agostini, Katerina M. Antoniou, Demosthenes Bouros, \\ Rachel C. Chambers, Vincent Cottin, Jim J. Egan, Bart N. Lambrecht, Rik Lories, \\ Helen Parfrey, Antje Prasse, Carlos Robalo-Cordeiro, Eric Verbeken, \\ Johny A. Verschakelen, Athol U. Wells and Geert M. Verleden
}

ABSTRACT: Pulmonary fibrosis is the end stage of many diffuse parenchymal lung diseases. It is characterised by excessive matrix formation leading to destruction of the normal lung architecture and finally death. Despite an exponential increase in our understanding of potentially important mediators and mechanisms, the delineation of primary pathways has proven to be elusive.

In this review susceptibility and injurious agents, such as viruses and gastro-oesophageal reflux, and their probable role in initiating disease will be discussed. Further topics that are elaborated are candidate ancillary pathways, including immune mechanisms, oxidative and endoplasmic reticulum stress, activation of the coagulation cascade and the potential role of stem cells. This review will try to provide the reader with an integrated view on the current knowledge and attempts to provide a road map for future research.

It is important to explore robust models of overall pathogenesis, reconciling a large number of clinical and scientific observations. We believe that the integration of current data into a "big picture" overview of fibrogenesis is essential for the development of effective antifibrotic strategies. The latter will probably consist of a combination of agents targeting a number of key pathways.

KEYWORDS: Coagulation, fibrosis, immunology, pathogenesis, pulmonary fibrosis

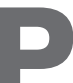

ulmonary fibrosis is the end stage of several diffuse parenchymal lung diseases (DPLDs), characterised by excessive matrix deposition and destruction of the lung architecture, finally leading to respiratory insufficiency. The most common form of pulmonary fibrosis, idiopathic pulmonary fibrosis (IPF), is a progressive disease with a 5-year survival rate of only $20 \%$, reflecting the lack of effective therapies. In the UK, $>3000$ people die each year from IPF and the incidence is increasing annually by $11 \%$ [1]. The aetiology of IPF remains poorly understood, although several risk factors and predisposing factors have been proposed, including cigarette smoking, viral infections and surfactant protein polymorphisms.

Histologically, IPF lungs have alternating regions of normal lung parenchyma, interstitial inflammation, fibrosis and honeycombing. The pathophysiological basis of IPF has been the subject of much debate over the last few decades. There is now growing evidence that IPF may represent a separate disorder in which fibrogenesis results, at least in part, from multi-focal epithelial micro-injury. Repeated injury to the alveolar epithelial cell (AEC) leads to apoptosis, which might lead to disordered epithelial-fibroblastic interactions and aberrant repair processes, finally resulting in fibrosis [2].

In this review, we focus on factors that make individuals susceptible to the process of progressive fibrosis, possible agents involved in repeated injury and important elements leading to aberrant repair and pulmonary fibrosis. In addition, emerging new findings will be discussed such as immunological processes, oxidative stress, endoplasmic reticulum (ER) stress, activation of the coagulation cascade and possibly alterations in the lymphatic vessels, and finally the role of stem cells.

\section{INITIATION OF FIBROSIS: PREDISPOSITION AND CAUSAL AGENTS}

Agents responsible for the initiation of the fibrotic process remain poorly defined. It is generally accepted that repeated injury is caused

\section{AFFILIATIONS}

For a full list of affiliations, refer to the Acknowledgements section.

CORRESPONDENCE

W.A. Wuyts

Dept of Respiratory Medicine

University Hospitals Leuven

Herestraat 49

3000 Leuven

Belgium

E-mail:wim.wuyts@uzleuven.be

Received:

May 082012

Accepted after revision:

Aug 242012

First published online:

Oct 252012 
by an interaction between genetic predisposition and injurious environmental agents.

\section{Genetic predisposition}

Although most DPLDs are sporadic diseases, examples of familial occurrence exist. Familial clustering of adult idiopathic interstitial pneumonias (IIP) suggests that genetic factors might play an important role in disease development. Interesting new findings include the characterisation of the ELMOD2 gene and the role of surfactant protein (SP)-C. Interestingly, genetic alterations have also been noted in sporadic IPF. ELMOD2 mRNA expression is significantly decreased in IPF lungs in comparison with healthy control subjects. ELMOD2 has been shown to be implicated in the regulation of interferon-related antiviral responses [3].

SP-C mutations have been frequently identified in children with severe idiopathic pneumonias [4]. Interestingly, these have been identified in $50 \%$ of these children with the other $50 \%$ having sporadic disease. In both children and adults, SP-C mutations are associated with the clinical entities of nonspecific interstitial pneumonia (NSIP), desquamative interstitial pneumonia (DIP) and pulmonary alveolar proteinosis. In a recent paper, VAN MOORSEL et al. [5] showed that five out of 20 unrelated patients with familial pulmonary fibrosis had a mutation in SP-C, with this particular mutation not found in sporadic disease.

The expression of mutations, such as SP-A and SP-C, in the alveolar epithelium and the identification of mutations affecting cell stability, such as telomerase (hTERT and hTER) mutations, leads to abnormal homeostasis of the alveolar epithelium. Mutations in SP-C and SP-A lead to dysfunction in alveolar Type-II (ATII) cells; probably as a result of misfolded proteins, ER retention and activation of unfolded protein responses [6]. A recent study has also shown that genetic abnormalities involving the airways might promote pulmonary fibrosis, as recently described for MUC5 [7]. A common variant in the putative promoter of MUC5B has been identified that is associated with the development of familial interstitial pneumonia and IPF. Subjects with IPF had significantly higher expression of MUC5B in the lungs than control subjects and MUC5B was expressed in the histological lesions of IPF. However, the exact link between MUC5B hypersecretion in the airways and the presence of disturbed alveolar epithelial homeostasis in pulmonary fibrosis is not yet clear.

\section{Injurious agents}

It is currently believed that that IPF results from the association of a genetic predisposition to abnormal epithelial cell regulation with environmental triggers, leading to the formulation of the "multiple hit hypothesis". In fibrotic diseases with known aetiology, fibrosis is driven by persisting injury that can be mediated by immune complexes, inhaled agents such as asbestos or direct toxic injury such as radiation or drugs. In IIPs, many potential causative agents have been proposed; the best known environmental factors are viruses and gastrooesophageal reflux (GOR).

\section{Viruses}

Viral infections have been postulated as initiators of fibrosis but are extremely difficult to study due to the variable sensitivity of respiratory sample cultures. With the development of PCR and other molecular detection methods, knowledge in this field has dramatically increased. Viruses implicated in the pathogenesis of IPF include Epstein-Barr virus (EBV), human herpes viruses 7 and 8, cytomegalovirus, hepatitis $C$ virus, herpes simplex virus, parvovirus B19 and torque teno virus. Another issue here is whether the presence of EBV and other viruses might merely reflect alveolar epithelial damage with an increased vulnerability to infection, although there is increasing evidence for a causal role of viruses in the initiation of fibrosis.

EBV has been the infection most widely identified in IPF, providing a potential source of repetitive cell injury, a central component of the current pathogenetic hypothesis [8]. TANG et al. [9] reported the presence of EBV, detected by real timePCR, in five $(62.5 \%)$ out of eight familial cases and in $16(64 \%)$ out of 25 sporadic cases of IPF. Although EBV usually infects the upper respiratory tract, it has also been shown to infect and replicate in the lower respiratory tract [10]. Both EBV protein and DNA expression have been identified in the lung tissue of IPF patients [11, 12]. In IPF biopsy samples, EBV gp340/220 and viral proteins, expressed during the lytic phase of EBV infection, have been localised to AEC [11]. The putative role of EBV in the development of IPF is supported by other observations. A poor prognosis in IPF patients has been associated with the expression of EBV latent membrane protein-1 in AEC [13] and with the replicative phase EBV infection. Clinical stability in two IPF patients following oral antiviral therapy has been reported [9]. Transforming growth factor (TGF)- $\beta 1$, a key pro-fibrotic cytokine implicated in IPF, is a potent growth inhibitor in most cells of epithelial origin [14]. EBV-infected cells exposed to TGF- $\beta 1$ show viral lytic phase activation and resistance to cell growth inhibition [15]. It has also been shown that EBV-AEC interactions might be involved in producing fibrotic scars, the pathological hallmarks of IPF. Moreover it is possible that bioactivity of EBV might induce epithelial mesenchymal transition (EMT).

Both genetic factors and virus-host interactions might be linked as demonstrated for the ELMOD2 gene. ELMOD2 is expressed by lung epithelial cells and alveolar macrophages. It regulates interferon-related antiviral responses and its expression is decreased in response to viral infection [3]. Interestingly epithelial cells and alveolar macrophages are the fundamental cell types infected by respiratory viruses. These findings strengthen the evidence for a strong correlation between virus-host interactions and genetic susceptibility factors.

\section{Gastro-oesophageal reflux}

From the 1970s onwards, an association between both GOR and hiatal herniation and DPLD has been recognised [16]. In IPF, the prevalence of GOR is estimated to be up to $90 \%$ [17]. Emerging data point to the potential role of chronic microaspiration due to the subclinical aspiration of small droplets, for which GOR is a risk factor. GOR and silent micro-aspiration are associated with several lung diseases, including posttransplantation rejection [18]. Another interesting study by TCHERAKIAN et al. [19] has provided evidence for a role of GOR in the pathogenesis of both IPF and acute exacerbations. These investigators studied a group of patients presenting with asymmetrical IPF. They found that GOR was present in 20 
(62.5\%) patients with asymmetrical IPF and in more symmetrical cases this was only present in $31.3 \%$ of patients. Patients with asymmetrical IPF were also reported to have much more acute exacerbations (46.9\%) than symmetrical cases $(17.2 \%)$ The authors conclude that asymmetry might highlight the role for an aetiological factor such as GOR or specific regional conditions in the development and/or progression of pulmonary fibrosis and acute exacerbation.

Other recent studies have reported on the association between acute exacerbations of IPF and GOR. Acute exacerbations are characterised by a histopathological pattern of diffuse alveolar damage superimposed on the underlying pattern of usual interstitial pneumonia (UIP). Pepsin is thought to play an important role in acute exacerbations as it has been found in the bronchoalveolar lavage (BAL) fluid of patients with acute exacerbations [20]. It was even found that elevated BAL pepsin was predictive of acute exacerbation status, mainly driven by the presence of a subgroup of cases (33\%) with markedly elevated pepsin levels.

Despite the compelling data supporting a correlation between GOR and IPF, this might alternatively be explained by the fact that GOR more often occurs in fibrotic lungs, or even results from the fibrotic changes in IPF, although this seems less likely at present.

\section{PROGRESSION OF FIBROSIS}

\section{Key cell types}

Currently, pulmonary fibrosis is regarded as a disease caused by repeated subclinical injury leading to epithelial damage and subsequent destruction of the alveolar-capillary basement membrane. This process initiates the infiltration of fibrotic cells and the activation of (myo)fibroblasts. In pulmonary fibrosis the normal resolution of inflammatory and mesenchymal cells through apoptosis and phagocytosis is dysregulated. This results in the destruction of the normal lung architecture and loss of function. In IPF this process leads to death with a median time of 3 years after diagnosis. These processes are summarised in figure 1.

\section{Alveolar epithelial cells}

Current in vitro and in vivo evidence suggests that AEC injury is a key process in the pathogenesis of IPF. An important observation in tissue of patients with IPF, first reported by KATZENSTEIN et al. [8], is hyperplasia of ATII and denudation of the alveolar epithelium within fibroblastic foci [21]. Moreover, as discussed earlier, mutations in genes that affect the regenerative capacity or that cause injury/apoptosis of ATII cells have been identified in familial forms of pulmonary fibrosis. [22]. In this process injury of the ATII is crucial, as it has been shown that in the lung of IPF patients $70-80 \%$ of the ATII stained positive for markers of apoptosis [23]. As shown very recently, targeted depletion of ATII in a mouse model of pulmonary fibrosis specifically links injury of ATII to the development of lung fibrosis [24, 25]. Enhanced fibroblast differentiation and collagen production has been shown in epithelial cell/fibroblast co-cultures in the presence of injury to the epithelial cell component [24]. Moreover mesenchymal cell survival is enhanced due to increased responsiveness to growth factors and resistance to apoptosis.
As classically described, injuries to ATII result in ineffective reconstitution of a normal epithelium and drive fibrosis by inducing the resident interstitial fibroblasts to differentiate into myofibroblasts [25, 26]. These are the key effector cells in pulmonary fibrosis and are characterised by the de novo expression of $\alpha$-smooth muscle actin, which is organised into functional stress fibres and confers contractile properties [27]. They localise to fibrotic foci and other sites of active fibrosis, and are responsible for the synthesis and deposition of extracellular matrix (ECM) and the resultant structural remodelling that leads to the loss of alveolar function. Current opinion suggests that myofibroblasts have at least three possible origins, although the relative contribution of each of these pathways to IPF is currently unknown. The most straightforward suggestion is that resident lung fibroblasts differentiate directly under the influence of the profibrotic microenvironment to form myofibroblasts. The second possibility is that epithelial cells undergo transdifferentiation to form fibroblasts/myofibroblasts by EMT. Epithelial cells lose their characteristic markers such as E-cadherin and zona occludens-1 and acquire mesenchymal markers, such as fibroblast-specific protein- 1 and $\alpha$-smooth muscle actin [28]. The third hypothesis suggests that myofibroblasts may be derived from circulating fibrocytes (collagen I+/CD34+/ $\mathrm{CD} 45 \mathrm{RO}+$ ) or other bone marrow-derived progenitor cells [29]. Attenuation of fibrocyte trafficking in mouse models directly correlates with a reduction in pulmonary fibrosis. The number of circulating fibrocytes has been recently shown to correlate with disease activity and progression [30]. Enhanced myofibroblast transformation and proliferation along with retarded apoptosis of mesenchymal cells are a major element in the relentless progression of fibrosis [31]. Furthermore, sustained matrix deposition or lack of matrix degradation further contributes to the destruction and replacement of normal alveolar tissue with dense fibrotic lesions.

\section{Key cytokines}

Recruitment of fibroblasts, fibroblast proliferation and overproduction of ECM is driven by a complex network of inflammatory cytokines, chemokines and cell types. Key cytokines and chemokines that induce a profibrotic milieu include tumour necrosis factor (TNF)- $\alpha$, TGF- $\beta$, monocyte chemotactic protein (MCP)1/CCL2, macrophage inhibitory protein (MIP)1 $\alpha /$ CCL3 and T-helper (Th)2-chemokines such as CCL17 CCL18 and CCL22 [32, 33]. TNF- $\alpha$ plays a central role in the stimulation of inflammatory responses, cell-cell adhesion and trans-endothelial migration, as well as in the early events leading to the cytokine and chemokine production cascade [34]. TNF- $\alpha$-transgenic mice develop a T-cell mediated alveolitis and subsequent fibrosis, while TNF- $\alpha$ knockout mice fail to develop fibrosis after treatment with bleomycin [35]. Despite these encouraging findings a study performed with etanercept in IPF patients showed no significant differences in efficacy end-points after treatment for 48 weeks [36].

TGF- $\beta$ is the most potent profibrotic mediator characterised to date and modulates lung fibrosis through the recruitment and activation of monocytes and fibroblasts, and the induction of ECM production. Lung fibroblast proliferation is an indirect effect of TGF- $\beta 1$ via the induction of fibroblast growth factor-2 


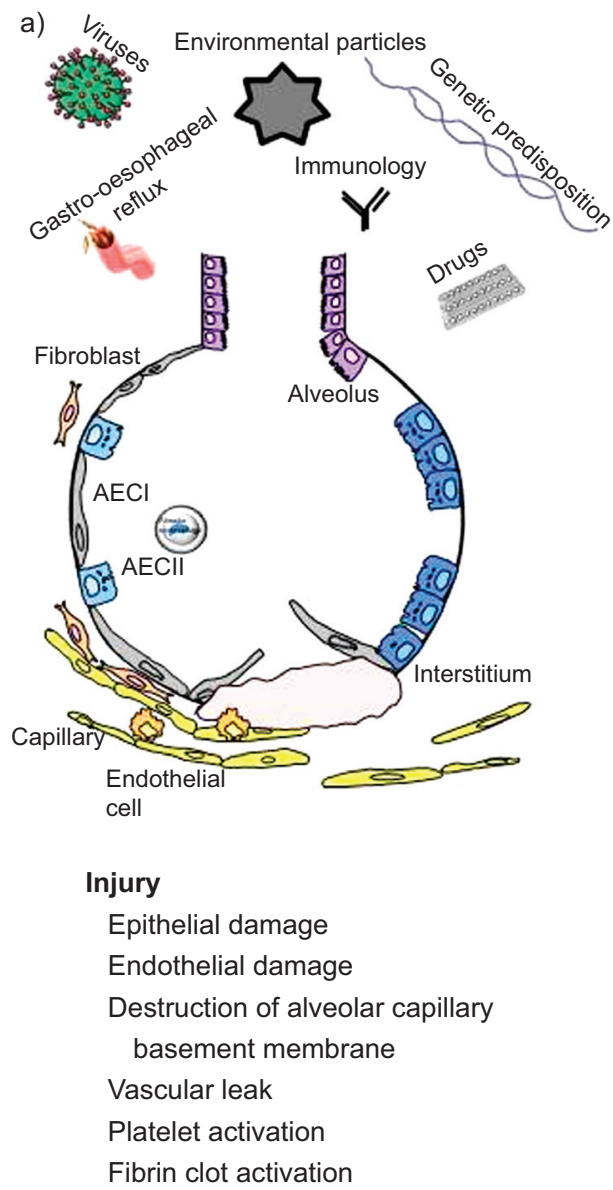

b)

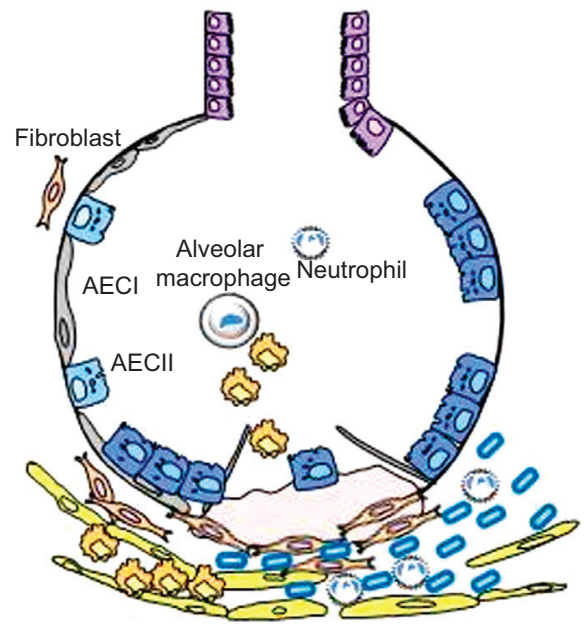

Epithelial-fibroblastic interaction

Release of profibrotic cytokines

(Myo)fibroblast recruitment,

proliferation and differentiation

Provisional matrix formation

Angiogenesis

Defective re-epithelialisation c)

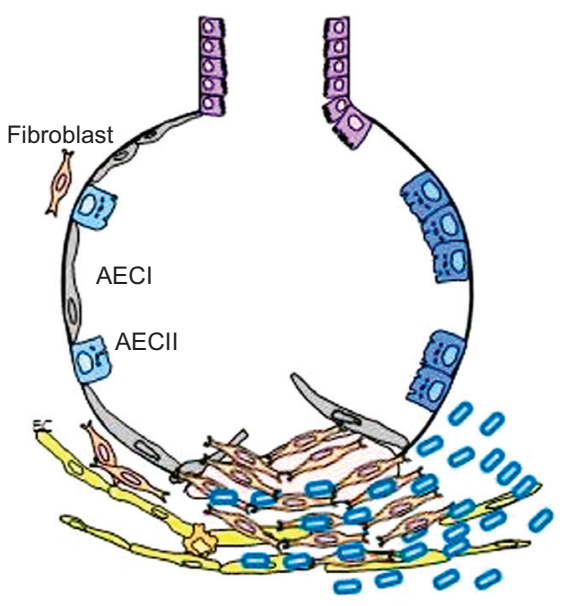

FIGURE 1. The major elements involved in induction and progression of fibrosis. a) The onset of fibrosis is characterised by both injury and susceptibility to the formation of progressive fibrosis. Many different injurious agents have been identified that lead to epithelial and endothelial damage, vascular leak and fibrin clot formation. b) This is followed by an abnormal repair process characterised by an abnormal re-epithelialisation, abundance of myofibroblasts and the formation of a collagen matrix. c) The process proceeds to excessive matrix formation leading to architectural distortion and finally death. ECM: extracellular matrix; AEC: alveolar epithelial cell.

and consequent activation of the mitogen-activated protein kinase (MAPK) pathway [37]. Furthermore, TGF- $\beta$ induces fibroblasts to differentiate into myofibroblasts. TGF- $\beta$ promotes ECM production by promoting ECM gene transcription, including collagen, fibronectin and proteoglycans and by suppressing the activity of matrix metalloproteinases, plasminogen activators and elastases, which results in the inhibition of collagen degradation [38].

MCP1/CCL2 and MIP1 $\alpha / C C L 3$ are pro-inflammatory chemokines responsible for monocyte recruitment. CCL2 and CCL3 are significantly upregulated both in BAL fluid from patients with IPF and in fibrotic lungs of mice treated with bleomycin [39]. MCP1 receptor CCR2-deficient mice are protected from bleomycin-induced pulmonary fibrosis due to an impairment in macrophage recruitment and function [40], fibrogenic cytokine expression and fibroblast responsiveness to TGF- $\beta$ [41].

Genomics is another field that has greatly contributed to our knowledge of fibrogenesis in recent years. Gene expression studies using lung tissue from patients with interstitial pneumonia have revealed that the molecular phenotype of IPF/UIP differs from that of NSIP, with the latter more closely resembling hypersensitivity pneumonitis [42]. Many genes significantly increased in fibrotic lungs encode proteins associated with ECM formation and degradation and proteins expressed by smooth muscle cells, including matrilysin, matrix metalloproteinase (MMP)7 [43], which is a metalloproteinase mediator of pulmonary fibrosis. It has become clear that MMPs exert pleiotropic effects, including proteolytic degradation of the ECM and in the processing of chemokines, cytokines and growth factors [44]. It might even be possible that enhanced MMP activity is, therefore, a mechanistic driver of progressive fibrosis in IPF. The molecular expression signature distinguishing progressive IPF from relatively stable IPF includes members of the MAPK-early growth response 1-heat shock protein 70 pathway that regulate smoke-induced inflammation. However, molecular and cellular functions, such as cell proliferation, migration, invasion and cell morphology, are the most over-represented in IPF and in tissue from patients with acute exacerbations of IPF [45], with gene expression analysis identifying epithelial injury and proliferation as key molecular events [46]. Collectively, these approaches indicate that in IPF lungs, genes and mechanisms that are associated with lung development (including the Wnt/ $\beta$-catenin pathway, epithelial cell plasticity and EMT) are activated, with a limited role for classical inflammation. 


\section{EMERGING NEW FINDINGS}

\section{Immunological mechanisms}

The inter-relationships between inflammation and fibrosis may be considered from various perspectives. Historical data in IPF suggested that an increased percentage of lymphocytes in BAL was associated with greater responsiveness to corticosteroid therapy and better survival [47]. BAL lymphocytosis was found to be associated with moderate-to-severe alveolar septal inflammation and a relative lack of honeycombing [48]. Later, with the emergence of NSIP as a distinct entity [49], it was suspected that many patients with a BAL lymphocytosis, formerly diagnosed with IPF, did in fact have NSIP. This is supported by the fact that three major East Asian studies showed truly striking lymphocyte levels in NSIP [50-52]. It is suggested from high-resolution computed tomography scan observations that those patients might have organising pneumonia/NSIP overlap.

It is now clear that the presence of a BAL lymphocytosis does not reliably discriminate between NSIP and UIP in individual patients [53], but there are data showing that an elevated lymphocyte count in BAL can help to differentiate between IPF and other DPLD. In this regard it is important to mention the study of OHSHIMO et al. [54] who reported that a cut-off level of $30 \%$ for lymphocytes in BAL demonstrated a favourable discriminative power for the diagnosis of IPF. Six $(8 \%)$ of the 74 patients with IPF based on clinical data and imaging showed a lymphocytosis of $\geqslant 30 \%$ in BAL. Their final diagnoses were idiopathic NSIP $(n=3)$ and hypersensitivity pneumonitis $(n=3)$.

\section{Interstitial pneumonias other than IPF}

Several studies of the reclassification of patients previously diagnosed with IPF, using current criteria, have demonstrated the impact of the histopathological pattern on long-term survival in patients with IIP. Idiopathic NSIP, DIP and cryptogenic organising pneumonia (COP) have a much better outcome than IPF $[55,56]$. As COP responds dramatically to oral corticosteroids and the 5-year survival in NSIP is $\sim 80 \%$ [57]. It can be concluded that inflammation, when indicative of COP, is clearly associated with a better reversibility and survival. COP is not considered as a fibrotic disease, although currently classified as an IIP.

The most frequent histological patterns in idiopathic disease, UIP and NSIP are frequently encountered in connective tissue disease-associated interstitial lung disease (CTD-ILD). Two key advances have been made in recent years in CTD-ILD. First, it has become clear that survival is better in CTD-ILD than in IPF [58], with the exception of lung disease in rheumatoid arthritis. Secondly, a number of histopathological features are more frequently observed in CTD than in IIP, including the pattern of NSIP and the presence of lymphoid aggregates and germinal centres in UIP (as well as more plasma cells), with a marginal relationship to survival [59]. Denser perivascular collagen deposition has also been reported in CTD.

However, it is not clear whether patients with a clinical diagnosis of IIP, in whom auto-antibodies are detected, have a different prognosis from those without antibodies. SONG et al. [59] have recently shown that the survival of patients with IPF/ UIP was not altered by the presence of auto-antibodies. Earlier studies have demonstrated that antinuclear antibodies may be found in up to $10-20 \%$ of patients with so-called IPF [60], with some subsequently reclassified as NSIP $[55,56]$. Antinuclear antibodies and, less frequently, anti-neutrophil cytoplasmic antibodies [61] may be found in IPF patients, although their significance is uncertain. The most frequent pathological pattern in CTD-ILD is NSIP, except for rheumatoid arthritis which is characterised by a higher frequency of UIP [62]. Moreover, it is clear that rheumatoid arthritis-associated UIP represents a possible exception to the improved survival seen in CTD-UIP compared to IPF/UIP [63]. PARK et al. [58] reported that rheumatoid arthritis-associated UIP tended to have a worse survival than non-rheumatoid arthritis CTD-associated NSIP or UIP. In a larger study of 82 patients with rheumatoid arthritisILD a worse survival was reported for patients with UIP pattern on high-resolution computed tomography. Moreover, they found that the estimated survival in rheumatoid arthritis-UIP did not differ significantly from that of IPF [64].

\section{Idiopathic pulmonary fibrosis}

Much information has been obtained from animal models of pulmonary fibrosis; however, it is important to bear in mind that these models are characterised by acute injury with an inflammatory response and do not truly reproduce all features central to the pathogenesis of IPF. Transfer to the lung epithelium of rodents of a variety of inflammatory cytokines and chemokines using replication-deficient adenoviral vectors resulted in marked increases in BAL inflammatory cells and tissue pneumonitis but without residual lung remodelling and fibrosis [65], with the notable exception of TGF- $\beta 1$ and cytokines such as interleukin (IL)-1 $\beta$ that lead to overexpression of active TGF- $\beta 1$. However, despite being poorly representative of human lung fibrosis, animal studies have collectively demonstrated a central role for TGF- $\beta 1$ as a key profibrotic mediator, and have also shown that induction of lung fibrosis is not always dependent on the degree of the inflammatory response [65].

Although overt inflammation is absent in the lungs of patients with IPF, inflammatory cytokines and chemokines (together with the coagulation cascade and eicosanoid lipid mediators) contribute to the interplay between the lung epithelium and fibroblasts, to the polarisation toward a Type-2 helper T-cell phenotype and to lymphoid neogenesis [66, 67], as well as to angiogenesis through an imbalance between angiogenic and angiostatic CXC chemokines [66], which will be discussed further in this review. Phenotypes of M1 and M2 macrophages have been recently described that support Th1 and Th2 responses, respectively.

Th2 responses characterise a number of pulmonary diseases, many of which culminate in tissue remodelling and fibrosis. A shift towards Th2 immune responses seems to predominate in IPF and promote fibrosis primarily via the secretion of profibrotic cytokines by the injured epithelium. In murine models of lung disease, animals with a response to tissue injury predominantly of the Th2 type, are more prone to pulmonary fibrosis after lung injury than those with a predominantly Th1 response $[67,68]$.

It has been shown that CCL18, a CC-chemokine that is regulated by Th2 cytokines, is associated with pulmonary 
fibrosis [33]. CCL17 and CCL22 and their receptor CCR4 have been found to be elevated in areas of fibrotic lung tissue as compared with normal pulmonary parenchyma [38, 69, 70]. In particular, CCR4 was found to be expressed mostly by macrophages present in fibrotic areas. Moreover it has been shown that neutralisation of CCL17 and CCL22 leads to a significant reduction of lung damage [71]. The Th2 immune response contributes to a failure of re-endothelialisation and re-epithelialisation, and leads to the release of profibrotic growth factors into the region of injury. These profibrotic cytokines initiate fibroblast migration to the site of injury and promote their proliferation and differentiation into myofibroblasts. Inflammation and tissue remodelling with pathologic fibrosis are common consequences of Th2 responses in the lung and other organs. IL-13 and TGF- $\beta 1$ are frequently coexpressed in these responses and are believed to play important roles in the pathogenesis of Th2-induced pathologies. This Th1/Th2 hypothesis has dominated our understanding of immune regulation, immune pathogenesis and host defence for decades, despite its flaws and inability to explain certain data regarding $\mathrm{T}$-cell mediated tissue damage.

An exciting finding is the fact that serum CCL18 concentrations have a predictive value in IPF [72]. CCL18 is a CCchemokine produced by human myeloid cells and has been described as a marker of the alternative activation of macrophages. Macrophage activation by Th2 cytokines induces a special phenotype, so-called "alternative activation". Alternatively, activated macrophages play a role in tissue repair processes such as wound healing and fibrosis. PRASSE et al. [72] showed a significantly higher mortality in patients with serum CCL18 concentrations $>150 \mathrm{ng} \cdot \mathrm{mL}^{-1}$. These findings are promising and should be implemented in the design of new clinical trials.

Recently, two novel CD4 T-cell subsets have been described that have revolutionised our understanding of immune function: the Th17 subset, which develops via different cytokine signals from those of Th1 and Th2 lineages, and the T-regulatory cells (Tregs). The Th17 subset is characterised by production of IL-17 and is involved in the pathogenesis of autoimmune tissue injury, including rheumatoid arthritis and allergen-specific responses. The central mediators generating the effector CD4+ Th17 subset are TGF- $\beta$, IL-23 and IL-17. Although Th17 cells are important in mucosal host defence, they can mediate immunopathological events. The IL-17 receptor is upregulated in the lungs of patients with hypersensitivity pneumonitis [73]. The key profibrotic cytokine in pulmonary fibrosis, TGF- $\beta$, has been found to be necessary for the differentiation of naive $\mathrm{CD}^{+} \mathrm{T}$-lymphocytes into Th17 cells in mice $[74,75]$, which could be another way TGF- $\beta$ promotes fibrosis. In a comprehensive review on mechanisms of fibrosis, WYNN and RAMALINGAM [76] suggest a role for the IL-1 $\beta$-IL-17-TGF- $\beta$ axis.

Tregs (CD4+CD25+foxp3+) represent the first well-defined expansion of the CD4+ T-cell functional range. Tregs suppress activation of the immune system and help maintain immune homeostasis and tolerance to self-antigens [77]. In case of a shortage of Tregs, the potential amplitude of Th1 and Th2 responses is increased resulting in excessive $\mathrm{T}$-cell immunity as associated with autoimmune disease, asthma and allergy.
An abundance of Tregs, however, will reduce the potential amplitude of Th1 and Th2 responses and may prevent adequate immunity to tumours and infectious diseases [77, 78]. A significant impairment of Treg suppressor function is evident in both the peripheral blood and BAL fluid in IPF patients. Identical findings were retrieved from lung tissue biopsies. Finally, an almost linear correlation of Tregs global impairment, both in functional and numerical level, with parameters of disease severity including pulmonary function tests, was demonstrated, suggesting that Tregs dysfunction may serve as a reliable prognosticator of disease progressiveness and treatment responsiveness and may provide clinicians with a novel tool for risk stratification of patients with IPF [79]. It can be surmised that the low numbers and the systemic and local Tregs dysfunction found in IPF patients may result in inefficient control of the pre-existing over exuberant Th2 response or contribute to a Th2 skew [74].

\section{Oxidative stress}

Oxidative stress is defined as an imbalance of the generation of reactive oxygen species (ROS) in excess of the capacity to neutralise them. Overproduction of ROS might lead to oxidative stress that causes tissue injury. Oxidative stress might promote a tissue microenvironment that favours fibrosis over regeneration. This might play a role in the development of apoptosis resistance of fibroblasts in IPF [77]. It has been shown that lung myofibroblasts secrete hydrogen peroxide, which may mediate fibrogenic effects and induce epithelial apoptosis [78]. Moreover neutrophils are a major source of matrix degrading enzymes, including neutrophil elastase, and contribute to oxidative stress. Modulating oxidative stress might be an interesting way of preventing further injury in an attempt of halting the process of pulmonary fibrosis. Interesting new data show that sivestat, a neutrophil elastase inhibitor, is able to alleviate bleomycin-induced pulmonary fibrosis [79]. Moreover, deficiency of the antioxidant glutathione (GSH) in epithelial lining fluid is thought to play a role in the initiation and progression of fibrosis [80]. The established link between oxidative stress and IPF provided a strong rationale for clinical trials, including the evaluation of $\mathrm{N}$-acetylcysteine (NAC), an antioxidant that acts as a precursor of GSH synthesis via the main metabolite cysteine. The IFIGENIA (Idiopathic pulmonary Fibrosis International Group Exploring NAC I Annual) trial examined the effect of high dose NAC in addition to corticosteroids and azathioprine. In this trial it was shown that the addition of NAC was able to slow down the progression of forced vital capacity and diffusing capacity of the lung for carbon monoxide decline in IPF [81]. However, the recent PANTHER (Prednisolone, Azathioprine and NAC: a study that evaluates response in IPF) trial has revealed that the combination of NAC with corticosteroids and azathioprine was associated with an increased risk of death and hospitalisation in comparison with placebo [82]. This has been considered new evidence against the initiation of this combination therapy for patients with IPF. One of the issues here is whether the individual characteristics of the IPF patients in this trial compare to past study populations [83], as the rate of death in the placebo arm in the PANTHER trial was only $2 \%$, which is lower than in other placebo-controlled trials in IPF. These results certainly warrant further detailed analysis. Furthermore, it is clear that the 
results of the continuing PANTHER IPF trial focussing on NAC monotherapy versus placebo are eagerly awaited.

\section{Endoplasmic reticulum stress}

ER stress is defined as being caused by conditions that disturb the processing and folding of proteins, which results in the accumulation of misfolded proteins in the ER and activation of the so called unfolded protein response (UPR) [84]. In the last few years, a vast amount of evidence has been provided that suggests a role for ER stress in IPF [23, 76, 85]. The initial observations were identified in AEC from families with SP-C mutation, but they have also been observed in non SP-Crelated familial IPF and even sporadic IPF [4, 23]. Secreted proteins are initially delivered to the ER as unfolded polypeptide chains. Thereafter they are properly folded into functional three dimensional conformations, assembled and glycosylated. When the cell is stressed, activation of the UPR can occur, failure of the UPR response, or too severe ER stress, might lead to AEC cell death through apoptosis [85]. Other studies suggest that ER stress can lead to a fibrotic AEC phenotype via an EMT mechanism, as this process may be involved in the regulation of cellular phenotypes [86]. In addition, viral infection initiates the production of large amounts of viral proteins, which may activate ER stress. Interestingly, it has been shown that herpes virus proteins that are expressed in AECs lining areas of fibrosis in IPF are colocalised with ER stress markers [85].

It is clear that aberrant protein processing is a potential target for future therapy, but more research in this intriguing field is needed. In particular, it is not clear what the link is between environmental insults and ER stress, or whether cell types other than AEC might also be involved in the ER stress-UPR axis. Moreover, there might be a link between surfactant proteins and ER stress. Patients with Hermansky-Pudlak syndrome, a rare autosomal recessive disorder associated with oculocutaneous albinism and haemorrhagic diathesis, sometimes develop interstitial pneumonia. It has recently been shown that the ER stress response seen in this disorder could be due to increasing amounts of accumulating SP-B/-C. This additional ER stress further contributes to AEC apoptosis [87].

\section{The vascular component and the coagulation cascade}

Aberrant vascular remodelling is crucial for the development and progression of IPF. A number of data indicate the importance of the altered balance between pro- (CXCL8, CXCL5 and CXCL12) and anti- (CXCL9, CXCL10 and CXCL11) angiogenic CXC chemokines in promoting aberrant neoangiogenesis and lung recruitment of circulating fibrocytes with the contribution of TGF- $\beta$.

The activation of the coagulation cascade is one of the earliest events initiated following tissue injury. The primary function of the coagulation cascade is to plug damaged blood vessels and prevent blood loss. However, it is increasingly recognised that the functions of the coagulation cascade extend beyond haemostasis and that this cascade plays a central role in influencing inflammatory and tissue repair programmes. The cellular responses of the coagulation cascade are principally executed by several coagulation proteinases, acting via specific cell-surface receptors, the proteinase-activated receptors (PARs). This family comprises four members (PAR1 to PAR4) but current evidence suggests a major role for the high-affinity thrombin receptor, PAR1, in influencing cellular responses in the context of lung injury. Activation of PAR1 on numerous lung cell types, including pulmonary epithelial cells, fibroblasts and macrophages, leads to the release and activation of potent pro-inflammatory and profibrotic mediators [88-90]. Moreover, PAR1 signalling in response to either thrombin or factor Xa on fibroblasts also promotes their proliferation and differentiation into matrix synthesising myofibroblasts [91-93].

Evidence that the coagulation cascade may be of pathophysiological significance has come from in vivo models of bleomycin-induced pulmonary fibrosis. Targeting the coagulation cascade in these models (using a variety of strategies, including direct thrombin inhibition, tissue factor pathway inhibition, intratracheal administration of activated protein $C$ and aerosolised heparin) led to a reduction in lung collagen accumulation and the development of fibrotic lesions [94]. A central role for the cell-mediated responses of the coagulation cascade in this model was provided by evidence that PAR1deficient mice are protected from experimental lung oedema, inflammatory cell recruitment and fibrosis [88, 95].

Current dogma assumes that coagulation zymogens are derived from the circulation and locally activated in response to injury via the extrinsic (tissue factor) coagulation pathway. However, it has been recently shown that coagulation factor $\mathrm{X}$ is locally upregulated in patients with IPF and in the murine model of bleomycin-induced lung injury, with both the bronchial and alveolar epithelium representing prominent cellular sources of this coagulation zymogen [96].

These findings herald a paradigm shift in our understanding of the tissue origin of excessive pro-coagulant signalling in lung disease and suggest that the epithelium is a major site for the initiation of such responses. In contrast to the promising preclinical studies with different anticoagulant strategies, a recent clinical trial suggests that systemic anticoagulant therapy using warfarin did not show any benefit in patients with progressive IPF. On the contrary, treatment with warfarin was associated with an increased risk of mortality in an IPF population who lacked other indications for anticoagulation [97]. It is not yet clear whether the effects of warfarin were entirely mediated via its anticoagulant effects or other vitamin K-dependent enzymes. It is also not known whether warfarin was effective at blocking coagulation within the intra-alveolar compartment. However, this trial raises the possibility that the coagulation cascade may also play protective roles in pulmonary fibrosis. Systemic anticoagulants may, therefore, not prove useful and future anticoagulant strategies may need to selectively target deleterious coagulation signalling responses within the intraalveolar compartment in IPF. Novel PAR1 antagonists are currently in phase III trials in the context of cardiovascular disease and may warrant further investigation in IPF [98].

\section{Lymphatics and pulmonary fibrosis}

In normal lung tissue, lymphatic vessels exist in close proximity to the airways and major blood vessels. Due to their thin wall structure, and the lack of reliable markers the knowledge of lymphatics in normal human lung is sparse. PUSZTASZERI et al. [99] found that D2-40, a marker of lymphatic endothelial cells, follows bronchovascular distribution and one 
study reported the absence of lymphatic vessels in the alveolar space [100]. Recently, Sozio et al. [101] have shown that lymphatic vessels extend within the lobule beyond respiratory bronchioles. Moreover, they provide evidence for heterogeneity of lymphatic vessels within the lobule, in comparison with lymphatics from other lung districts [101]. In pig, lung lymphatic vessels were not found in the parenchyma [102]. An interesting finding has been reported in hypoxia induced alveolar damage in a rat model [103]. The authors found perialveolar lymphatic vessels, which resolved over a 14-day period.

In IPF the data on lymphangiogenesis are sparse. It is well known that mediastinal lymphadenopathy is present in a significant amount of patients with IPF [104, 105]. In the study of EL-CHEMALY et al. [106], lymphatic vessels in IPF lungs were in close proximity to alveolar spaces, even in those areas with a well-preserved architecture. Moreover lymphatic vessels were present throughout the fibrotic tissue and in close proximity to the main bronchovascular tree. Within fibroblastic foci, there were no lymphatic vessels observed, while lymphatics could be seen at their periphery. In diffuse alveolar damage, intraalveolar lymphangiogenesis is a key element of the fibrotic process [100].

However, there are also data suggesting that both the subpleural and the interlobular lymphatics in IPF lungs are significantly decreased in the severe fibroconnective lesions, with rare lymphangiogenesis [107]. Moreover, destruction of the subpleural lymphatics and apoptosis of lymphatic endothelial cells have been observed in IPF patients. The disappearance of the subpleural and interlobular lymphatics in IPF lungs, along with poor lymphangiogenesis, may significantly impair alveolar clearance and prolong exposure to damaging mediators in the interstitium, enhancing their influence on fibrogenesis. This is a particularly attractive model for acute exacerbations, in which the coagulation cascade and lymphatic disruption may synergise in a most damaging fashion.

\section{Stem cells in pulmonary fibrosis}

A broad range of cells have been shown to exhibit the capacity to differentiate into lung cells. These range from lung progenitor cells, circulating cells, mesenchymal stem cells (MSCs), induced pluripotent stem cells, placental stem cells and human embryonic stem cells (HUES). The common strategy for treatment of fibrotic lung diseases is to replace damaged epithelial and endothelial cells and restore normal repair. MSCs and placental stem cells have both been shown to express markers of lung epithelium following injection into animal models of lung diseases [108], and embryonic stem cells show features of lung epithelium in vitro [109].

Recently, a study from SPITALIERI et al. [110] has shown that HUES-3 can differentiate in vitro in ATII cells. Furthermore, transplantation of HUES-3-ATII into silica damaged mice significantly reduced markers of inflammation and fibrosis. In addition, lung engraftment of MSCs immediately after bleomycin-induced injury have been shown to have beneficial effects associated with decreased collagen deposition, fibrosis and MMP levels [111].

At present there are almost no clinical trials investigating this highly challenging issue of stem cells in pulmonary fibrosis. It is clear that this is an exciting new potential therapy that urgently requires further investigation.

\section{CONCLUSION}

After many years of relative standstill in pulmonary fibrosis, with treatments confined to anti-inflammatory agents, knowledge has exponentially increased in the last decade. New concepts have resulted in a major change in the understanding of this intriguing disease. In the current paradigm, pulmonary fibrosis is considered to be the result of multifactorial disease processes. Generally, it is thought that in the genetically susceptible host, exposure to injurious agents may result in phenotypic alterations of structural cells. This leads to aberrant cell interactions and finally fibrosis. Despite the unravelling of several core mechanisms of fibrosis, emerging data have generated new areas of interest.

One of the most challenging areas is the field of genetics. There is a better understanding of the factors involved in susceptibility to develop pulmonary fibrosis. Interesting new findings are ELMOD2, SP-C mutations, hTER and hTERT, and also MUC5B, although the exact linkage between MUC5B hypersecretion in the airways and the process of fibrosis is not yet clear. Also of major interest, is to further explore the link between genetic abnormalities and the pattern and severity of fibrosis in the individual patient. Furthermore, there is emerging evidence for a role of injurious agents. Promising new data have been published on the role of viruses in the initiation and propagation of the fibrotic process, especially for EBV. EBV has indeed been detected in IPF lungs, and although there are some ideas on the mechanism of EBV induced fibrosis, many questions still remain unanswered. GOR seems to be another injurious agent, although until now the effect of treatment of GOR on the formation of fibrosis was not clear at all. Whether non-acid reflux is involved, and to what extend this can be treated, is still largely unknown. In addition, it is clear that the link between injurious agents and genetic susceptibility needs more clarification.

The basic mechanisms of fibrosis have become much clearer, such as the important role of ATII cells and myofibroblasts, and also many elements of the cytokine network have been further explored. Other emerging areas are the role of immunological mechanisms on the initiation and modulation of the fibrotic process. Immunology has recently been boosted by new discoveries. One of the most exciting new findings is the fact that serum CCL18, a marker for alternative activated macrophages, has a prognostic value in IPF. This points to a possible role of macrophages in IPF. Until now these cells have been relatively neglected in IPF. Although functional CD4+ T-cell development has been dominated by the Th1-Th2 paradigm, the discovery of the Th17 pathway and its relationship with Tregs (fig. 1) opens a new and fascinating era in our understanding of adaptive immune regulation. This will certainly result in novel and more effective therapeutic approaches in IPF as in a number of autoimmune and inflammatory diseases.

Oxidative stress remains a challenging area as the role of antioxidative agents in the treatment of IPF is still not elucidated. The field of ER stress is much newer and many interesting findings have been published in the past few years. The relationship between ER stress and other immunological active and structural 
cells, especially ATII, warrants further investigation. The knowledge of the role of the vascular compartment is rather poor, but will become increasingly important with the role of the different coagulation pathways now well established. The next step will be an attempt to come to an integrated vision on the role of those pathways and the fibrotic process and how new therapeutics can be developed based on these new findings.

A complete new area is that of pulmonary lymphatics. Further development of this intriguing field will probably lead to the discovery of new pathways in the process of fibrosis. Last but not least, the development of stem cells from different sources is undoubtedly connected with a wide range of new possibilities. In vitro, there are many promising observations, but the usefulness of the different types of stem cells to stop the relentless process of fibrosis and to restore appropriate repair still needs to be explored.

Although seemingly the major steps and key players in the formation of fibrosis have been identified, it remains nevertheless important to explore robust models of overall pathogenesis, reconciling a large number of clinical and scientific observations. We believe that the integration of current data into a "big picture" overview of fibrogenesis is essential for the development of effective anti-fibrotic strategies.

\section{STATEMENT OF INTEREST}

Conflict of interest information can be found alongside the online version of this article at www.erj.ersjournals.com

\section{ACKNOWLEDGEMENTS}

The author's affiliations are as follows. Wim A. Wuyts: Laboratory of Pneumology, Dept of Respiratory Medicine, Katholieke Universiteit Leuven, Leuven and Dept of Respiratory Medicine, University Hospitals Leuven, Leuven, Belgium. Carlo Agostini: Dept of Clinical and Experimental Medicine, Clinical Immunology, Padua University, Padua, Italy. Katerina M. Antoniou: Dept of Thoracic Medicine, University of Crete, Heraklion, Greece. Demosthenes Bouros: Dept of Pneumonology, Medical School, Democritus University of Thrace, Alexandroupolis, Greece. Rachel C. Chambers: Centre for Inflammation and Tissue Repair, University College London, London, UK. Vincent Cottin: Hospices civils de Lyon, Hôpital Louis Pradel, Service de Pneumologie, Centre de Référence des Maladies Pulmonaires Rares, Université Lyon I, Lyon, France. Jim J. Egan: Mater Misericordiae University Hospital and St Vincent's University Hospital, University College, Dublin, Ireland. Bart N. Lambrecht: Dept of Molecular Biomedical Research, VIB, Gent, Belgium. Rik Lories: Laboratory for Skeletal Development and Joint Disorders, Division of Rheumatology, Dept of Musculoskeletal Sciences, Katholieke Universiteit, Leuven, Belgium. Helen Parfrey: Respiratory Medicine Division, Dept of Medicine, University of Cambridge and Papworth Hospital, Cambridge, UK. Antje Prasse: Dept of Pneumology, University Medical Center Freiburg, Freiburg, Germany. Carlos Robalo-Cordeiro: Dept of Pulmonology, University Hospital of Coimbra, Coimbra, Portugal. Eric Verbeken: Dept of Pathology, UZ Leuven, Leuven, Belgium. Johny A. Verschakelen: Dept of Radiology, UZ Leuven, Leuven, Belgium. Athol U. Wells: Interstitial Lung Disease Unit, Royal Brompton Hospital, London, UK. Geert M. Verleden: Lung Transplantation Unit, University Hospital Gasthuisberg, Leuven, Belgium.

\section{REFERENCES}

1 Gribbin J, Hubbard RB, Le Jeune I, et al. Incidence and mortality of idiopathic pulmonary fibrosis and sarcoidosis in the UK. Thorax 2006; 61: 980-985.
2 Selman M, Pardo A. Idiopathic pulmonary fibrosis: an epithelial/fibroblastic cross-talk disorder. Respir Res 2002; 3: 3.

3 Pulkkinen V, Bruce S, Rintahaka J, et al. ELMOD2, a candidate gene for idiopathic pulmonary fibrosis, regulates antiviral responses. FASEB J 2010; 24: 1167-1177.

4 Nogee LM, Dunbar AE, Wert SE, et al. A mutation in the surfactant protein $\mathrm{C}$ gene associated with familial interstitial lung disease. N Engl J Med 2001; 344: 573-579.

5 Van Moorsel CHM, Van Oosterhout MFM, Barlo NP, et al. Surfactant protein $\mathrm{C}$ mutations are the basis of a significant portion of adult pulmonary fibrosis in a Dutch Cohort. Am J Respir Crit Care Med 2010; 182: 1419-1425.

6 Chibbar R, Shih F, Baga M, et al. Nonspecific interstitial pneumonia and usual interstitial pneumonia with mutation in surfactant protein C in familial pulmonary fibrosis. Mod Pathol 2004; 17: 973-980.

7 Seibold MA, Wise AL, Speer MC, et al. A Common MUC5B promoter polymorphism and pulmonary fibrosis. N Engl J Med 2011; 364: 1503-1512.

8 Katzenstein ALA, Myers JL. Idiopathic pulmonary fibrosis. Clinical relevance of pathologic classification. Am J Respir Crit Care Med 1998; 157: 1301-1315.

9 Tang YW, Johnson JE, Browning PJ, et al. Herpes virus DNA is consistently detected in lungs of patients with idiopathic pulmonary fibrosis. J Clin Microbiol 2003; 41: 2633-2640.

10 Lung ML, Lam WK, So SY, et al. Evidence that respiratory tract is major reservoir for Epstein-Barr virus. Lancet 1985; 1: 889-892.

11 Egan JJ, Stewart JP, Hasleton PS, et al. Epstein-Barr virus replication within pulmonary epithelial cells in cryptogenic fibrosing alveolitis. Thorax 1995; 50: 1234-1239.

12 Stewart JP, Egan JJ, Ross AJ, et al. The detection of Epstein-Barr virus DNA in lung tissue from patients with idiopathic pulmonary fibrosis. Am J Respir Crit Care Med 1999; 159: 1336-1341.

13 Tsukamoto K, Hayakawa $\mathrm{H}$, Sato A, et al. Involvement of Epstein-Barr virus latent membrane protein 1 in disease progression in patients with idiopathic pulmonary fibrosis. Thorax 2000; 55: 958-961.

14 Sporn $M B$, Roberts $A B$. Transforming growth factor- $\beta$ : recent progress and new challenges. J Cell Biol 1992; 119: 1017-1021.

15 Fukuda M, Ikuta K, Yanagihara K, et al. Effect of transforming growth factor- $\beta 1$ on the cell growth and Epstein-Barr virus reactivation in EBV-infected epithelial cell lines. Virology 2001; 288: 109-118.

16 Pearson JE, Wilson RS. Diffuse pulmonary fibrosis and hiatus hernia. Thorax 1971; 26: 300-305.

17 Raghu G, Freudberger TD, Yang S, et al. High idiopathic pulmonary fibrosis. Eur Respir J 2006; 27: 136-142.

18 Sweet MP, Patti MG, Hoopes C, et al. Gastroeosophageal reflux and aspiration in patients with advanced lung disease. Thorax 2009; 64: 167-173.

19 Tcherakian C, Cottin V, Brillet PY, et al. Progression of idiopathic pulmonary fibrosis: lessons from asymmetrical disease. Thorax 2011; 66: 226-231.

20 Lee JS, Song JW, Wolters PJ, et al. Bronchoalveolar lavage pepsin in acute exacerbation of idiopathic pulmonary fibrosis. Eur Respir J 2012; 39: 352-358.

21 Kasper M, Haroske G. Alterations in the alveolar epithelium after injury leading to pulmonary fibrosis. Histol Histopathol 1996; 11: $463-483$

22 Thomas AQ, Lane K, Phillips J 3rd, et al. Heterozygosity for a surfactant protein $\mathrm{C}$ gene mutation associated with usual interstitial pneumonitis and cellular nonspecific interstitial pneumonitis in one kindred. Am J Respir Crit Care Med 12002, 65: 1322-1328.

23 Korfei M, Ruppert C, Mahavadi P, et al. Epithelial endopalmic reticulum stress and apoptosis in sporadic idiopathic pulmonary fibrosis. Am J Respir Crit Care Med 2008; 178: 838-846. 
24 Morishima Y, Nomura A, Uchida Y, et al. Triggering the induction of myofibroblast and fibrogenesis by airway epithelial shedding. Am J Respir Cell Mol Biol 2011; 24: 1-11.

25 Sisson TH, Mendez M, Choi K, et al. Targeted Injury of type II alveolar epithelial cells induces pulmonary fibrosis. Am J Respir Crit Care 2009; 164: 1605.

26 Selman M, Pardo A. Role of epithelial cells in idiopathic pulmonary fibrosis: from innocent targets to serial killers. Proc Am Thorac Soc 2006; 3: 364-372.

27 Scotton CJ, Chambers RC. Molecular targets in pulmonary fibrosis: the myofibroblast in focus. Chest 2007; 132: 1311-1321.

28 Willis BC, Borok Z. TGF- $\beta$-induced EMT: mechanisms and implications for fibrotic lung disease. Am J Physiol Lung Cell Mol Physiol 2007; 293: L525-L534.

29 Phillips RJ, Burdick MD, Hong K, et al. Circulating fibrocytes traffic to the lungs in response to CXCL12 and mediate fibrosis. J Clin Invest 2004; 114: 438-446.

30 Moeller A, Gilpin SE, Ask K, et al. Circulating fibrocytes are an indicator of poor prognosis in idiopathic pulmonary fibrosis. Am J Respir Crit Care Med 2009; 179: 588-594.

31 Coward WR, Saini G, Jenkins G. The pathogenesisi of idiopathic pulmonary fibrosis. Ther Adv Respir Dis 2010; 4: 367-388.

32 Agostini C, Gurrieri C. Chemokine/cytokine cocktail in idiopathic pulmonary fibrosis. Proc Am Thorac Soc 2006; 3: 357-363.

33 Prasse A, Pechkovsky DV, Toews GB, et al. A vicious circle of alveolar macrophages and fibroblasts perpetuates pulmonary fibrosis via CCL18. Am J Respir Crit Care Med 2006; 173: 781-792.

34 Zhang K, Gharaee-Kermani M, McGarry B, et al. TNF- $\alpha$ mediated lung cytokine networking and eosinophil recruitment in pulmonary fibrosis. J Immunol 1997; 158: 954-959.

35 Miyazaki Y, Araki K, Vesin C, et al. Expression of a tumor necrosis factor- $\alpha$ transgene in murine lung causes lymphocytic and fibrosing alveolitis. A mouse model of progressive pulmonary fibrosis. J Clin Invest 1995; 96: 250-259.

36 Raghu G, Brown KK, Costabel U, et al. Treatment of idiopathic pulmonary fibrosis with etanercept: an exploratory, placebocontrolled trial. Am J Respir Crit Care Med 2008; 178: 948-955.

37 Khalil N, Xu YD, O'Connor R, et al. Proliferation of pulmonary interstitial fibroblasts is mediated by transforming growth factor-beta1-induced release of extracellular fibroblast growth factor-2 and phosphorylation of p38 MAPK and JNK. J Biol Chem 2005; 280: 43000-43009.

38 Ruiz V, Ordonez RM, Berumen J, et al. Unbalanced collagenases/ TIMP-1 expression and epithelial apoptosis in experimental lung fibrosis. Am J Physiol Lung Cell Mol Physiol 2003; 285: L1026-L1036.

39 Zhang K, Gharaee-Kermani M, Jones ML, et al. Lung monocyte chemoattractant protein-1 gene expression in bleomycin-induced pulmonary fibrosis. J Immunol 1994; 153: 4733-4741.

40 Okuma T, Terasaki Y, Kaikita K, et al. C-C chemokine receptor 2 (CCR2) deficiency improves bleomycin-induced pulmonary fibrosis by attenuation of both macrophage infiltration and production of macrophage-derived matrix metalloproteinases. J Pathol 2004; 204: 594-604.

41 Gharaee-Kermani M, McCullumsmith RE, Charo IF, et al. CCchemokine receptor 2 required for bleomycin-induced pulmonary fibrosis. Cytokine 2003; 24: 266-276.

42 Selman M, Pardo A, Barrera L, et al. Gene expression profiles distinguish idiopathic pulmonary fibrosis from hypersensitivity pneumonitis. Am J Respir Crit Care Med 2006; 173: 188-198.

43 Zuo F, Kaminski N, Eugui E, et al. Gene expression analysis reveals matrilysin as a key regulator of pulmonary fibrosis in mice and humans. Proc Natl Acad Sci USA 2002; 99: 6292-6297.

44 Dancer RCA, Wood AM, Thickett DR. Metalloproteinases in idiopathic pulmonary fibrosis. Eur Respir J 2011; 38: 1461-1467.

45 Selman M, Carrillo G, Estrada A, et al. Accelerated variant of idiopathic pulmonary fibrosis: clinical behavior and gene expression pattern. PLoS One 2007; 2: e482.
46 Konishi K, Gibson KF, Lindell KO, et al. Gene expression profiles of acute exacerbations of idiopathic pulmonary fibrosis. Am J Respir Crit Care Med 2009; 180: 167-175.

47 Rudd RM, Haslam PL, Turner-Warwick M. Cryptogenic fibrosing alveolitis. Relationships of pulmonary physiology and bronchoalveolar lavage to response to treatment and prognosis. Am Rev Respir Dis 1981; 124: 1-8.

48 Watters LC, Schwarz MI, Cherniack RM, et al. Idiopathic pulmonary fibrosis. Pretreatment bronchoalveolar lavage cellular constituents and their relationships with lung histopathology and clinical response to therapy. Am Rev Respir Dis 1987; 135: 696-704.

49 Katzenstein ALA, Fiorelli RF. Nonspecific interstitial pneumonia/fibrosis. Histological features and clinical significance. Am J Surg Pathol 1994; 18: 136-147.

50 Ryu YJ, Chung MP, Han J, et al. Bronchoalveolar lavage in fibrotic idiopathic interstitial pneumonias. Respir Med 2007; 101: 655-660.

51 Park CS, Jeon JW, Park SW. Nonspecific interstitial pneumonia/ fibrosis: clinical manifestations, histologic and radiologic features. Korean J Intern Med 1996; 11: 122-132.

52 Nagai S, Kitaichi M, Itoh $\mathrm{H}$, et al. Idiopathic nonspecific interstitial pneumonia/fibrosis: comparison with idiopathic pulmonary fibrosis and BOOP. Eur Respir J 1998; 12: 1010-1019.

53 Veeraraghavan S, Latsi PI, Wells AU, et al. BAL findings in idiopathic nonspecific interstitial pneumonia and usual interstitial pneumonia. Eur Respir J 2003; 22: 239-244.

54 Ohshimo S, Bonella F, Cui A, et al. Significance of bronchoalveolar lavage for the diagnosis of idiopathic pulmonary fibrosis. Am J Respir Crit Care Med 2009; 179: 1043-1047.

55 Bjoraker JA, Ryu JH, Edwin MK, et al. Prognostic significance of histopathologic subsets in idiopathic pulmonary fibrosis. Am J Respir Crit Care Med 1998; 157: 199-203.

56 Daniil ZD, Gilchrist FC, Nicholson AG, et al. A histological pattern of nonspecific interstitial pneumonia is associated with a better prognosis than usual interstitial pneumonia in patients with cryptogenic fibrosing alveolitis. Am J Respir Crit Care Med 1999; 160: 899-905.

57 Travis WD, Hunninghake G, King TE Jr, et al. Idiopathic nonspecific interstitial pneumonia: report of an American Thoracic Society project. Am J Respir Crit Care Med 2008; 177: 1338-1347.

58 Park JH, Kim DS, Park IN, et al. Prognosis of fibrotic interstitial pneumonia: idiopathic versus collagen vascular disease-related subtypes. Am J Respir Crit Care Med 2007; 175: 705-711.

59 Song JW, Do KH, Kim MY, et al. Pathologic and radiologic differences between idiopathic and collagen vascular diseaserelated usual interstitial pneumonia. Chest 2009; 136: 23-30.

60 Strange C, Highland KB. Interstitial lung disease in the patient who has connective tissue disease. Clin Chest Med 2004; 25, 549 559: vii.

61 Flaherty KR, Travis WD, Colby TV, et al. Histopathologic variability in usual and nonspecific interstitial pneumonias. Am J Respir Crit Care Med 2001; 164: 1722-1727.

62 Lee HK, Kim DS, Yoo B, et al. Histopathologic pattern and clinical features of rheumatoid arthritis-associated interstitial lung disease. Chest 2005; 127: 2019-2027.

63 de Lauretis A, Veeraraghavan S, Renzoni E. Review series: aspects of interstitial lung disease: connective tissue diseaseassociated interstitial lung disease: how does it differ from IPF? How should the clinical approach differ? Chron Respir Dis 2011; 8: 53-82.

64 Kim EJ, Elicker BM, Maldonado F, et al. Usual interstitial pneumonia in rheumatoid arthritis-associated interstitial lung disease. Eur Respir J 2010; 35: 1322-1328.

65 Hardie WD, Glasser SW, Hagood JS. Emerging concepts in the pathogenesis of lung fibrosis. Am J Pathol 2009; 175: 3-16. 
66 Keane MP. The role of chemokines and cytokines in lung fibrosis. Eur Respir Rev 2008; 17: 151-156.

67 Strieter RM. Pathogenesis and natural history of usual interstitial pneumonia: the whole story or the last chapter of a long novel. Chest 2005; 128: Suppl. 1, 526S-532S.

68 Gross TJ, Hunninghake GW. Idiopathic pulmonary fibrosis. $N$ Engl J Med 2001; 345: 517-525.

69 Steinman L. A brief history of $\mathrm{T}(\mathrm{H}) 17$, the first major revision in the $\mathrm{T}(\mathrm{H}) 1 / \mathrm{T}(\mathrm{H}) 2$ hypothesis of $\mathrm{T}$ cell-mediated tissue damage. Nat Med 2007; 13: 139-145.

70 Yogo $\mathrm{Y}$, Fujishima S, Inoue $\mathrm{T}$, et al. Macrophage derived chemokine (CCL22), thymus and activation-regulated chemokine (CCL17), and CCR4 in idiopathic pulmonary fibrosis. Respir Res 2009; 10: 80.

71 Belperio JA, Dy M, Murray L, et al. The role of the Th2 CC chemokine ligand CCL17 in pulmonary fibrosis. J Immunol 2004; 173: 4692-4698.

72 Prasse A, Probst C, Bargagli E, et al. Serum CC-Chemokine ligand 18 concentration predicts outcome in idiopathic pulmonary fibrosis. Am J Respir Crit Care Med 2009; 179: 717-723.

73 Joshi AD, Fong DJ, Oak SR, et al. Interleukin-17-mediated immunopathogenesis in experimental hypersensitivity pneumonitis. Am J Respir Crit Care Med 2009; 179: 705-716.

74 Belghith M, Bluestone JA, Barriot S, et al. TGF-beta-dependent mechanisms mediate restoration of self-tolerance induced by antibodies to CD3 in overt autoimmune diabetes. Nat Med 2003; 9: 1202-1208.

75 Lambeth JD. Nox enzymes, ROS and chronic disease: an example of antagonistic pleiotropy. Free Radic Biol Med 2007; 43: 332-347.

76 Wynn TA, Ramalingam TR. Mechanisms of fibrosis: therapeutic translation or fibrotic disease. Nat Med 2012; 18: 1029-1040.

77 Wang E. Senescent human fibroblasts resit programmed cell death and failure to suppress bcl2 is involved. Cancer Res 1995; 55: 2284-2292.

78 Waghray M, Cui Z, Horowitz JC, et al. Hydrogen peroxide is a diffusible paracrine signal for the induction of epithelial cell death by activated myofibroblasts. FASEB J 2005; 19: 854-856.

79 Takemasa A, Ishii Y, Fukuda TA. Neutrophil elastase inhibitor prevents bleomycin-induced pulmonary fibrosis in mice. Eur Respir J 2012; 40: 1475-1482.

80 Cantin AM, Hubbard RC, Crystal RG. Glutathione deficiency in the epithelial lining fluid of the lower respiratory tract in idiopathic pulmonary fibrosis. Am Rev Respir Dis 1989; 139: 370-372.

81 Demedts M, Behr J, Buhl R, et al. High-dose $N$-acetylcysteine in idiopathic pulmonary fibrosis. N Engl J Med 2005; 353: 2229-2242.

82 Idiopathic Pulmonary Fibrosis Clinical Research Network, Raghu G, Anstrom KJ, et al. Prednisone, azathioprine, and Nacetylcysteine for pulmonary fibrosis. N Engl J Med 2012; 366: 1968-1977.

83 Wells AU, Behr J, Costabel U, et al. Triple therapy in idiopathic pulmonary fibrosis: an alarming press release. Eur Respir J 2012; 39: 805-806

84 Schroder M, Kaufman RJ. The mammalian unfolded protein response. Annu Rev Biochem 2005; 74: 739-789.

85 Lawson WE, Crossno PF, Polosukhin VV, et al. Endoplasmic reticulum stress in alveolar epithelial cells is prominent in IPF: association with altered surfactant protein processing and herpes virus infection. Am J Physiol Lung Cell Mol Physiol 2008; 294: L1119-L1126.

86 Tanjore H, Cheng DS, Degryse AL, et al. Alveolar epithelial cells undergo epithelial-to mesenchymal transition in response to endoplasmic reticulum stress. J Biol Chem 2001; 286: 30972-30980.

87 Mahavadi P, Korfei M, Henneke I, et al. Epithelial stress and aopotosis underlie Hermansky-Pudlak syndrome-associated interstitial pneumonia. Am J Respir Crit Care Med 2010; 182: 207-219.

88 Chambers RC, Leoni P, Blanc-Brude OP, et al. Thrombin is a potent inducer of connective tissue growth factor production via proteolytic activation of protease-activated receptor-1. J Biol Chem 2000; 275: 35584-35591.

89 Jenkins RG, Su X, Su G, et al. Ligation of protease-activated receptor 1 enhances $\alpha(v) \beta 6$ integrin-dependent TGF- $\alpha$ activation and promotes acute lung injury. J Clin Invest 2006; 116: 1606-1614.

90 Mercer PF, Johns RH, Scotton CJ, et al. Pulmonary epithelium is a prominent source of proteinase-activated receptor-1-inducible CCL2 in pulmonary fibrosis. Am J Respir Crit Care Med 2009; 179: 414-425.

91 Blanc-Brude OP, Archer F, Leoni P, et al. Factor Xa stimulates fibroblast procollagen production, proliferation, and calcium signaling via PAR1 activation. Exp Cell Res 2005; 304: 16-27.

92 Chambers RC, Dabbagh K, McAnulty RJ, et al. Thrombin stimulates fibroblast procollagen production via proteolytic activation of protease-activated receptor 1. Biochem J 1998; 333: 121-127.

93 Bogatkevich GS, Tourkina E, Silver RM, et al. Thrombin differentiates normal lung fibroblasts to a myofibroblast phenotype via the proteolytically activated receptor- 1 and a protein kinase C-dependent pathway. J Biol Chem 2001; 276: 45184-45192.

94 Chambers RC. Procoagulant signalling mechanisms in lung inflammation and fibrosis: novel opportunities for pharmacological intervention? Br J Pharmacol 2008; 153: Suppl. 1, S367-S378.

95 Howell DC, Johns RH, Lasky JA, et al. Absence of proteinaseactivated receptor-1 signaling affords protection from bleomycin-induced lung inflammation and fibrosis. Am J Pathol 2005; 166: 1353-1365.

96 Scotton CJ, Krupiczojc MA, Königshoff M, et al. Increased local expression of coagulation factor $\mathrm{X}$ contributes to the fibrotic response in human and murine lung injury. J Clin Invest 2009; 119: 2550-2563.

97 Noth I, Anstrom KJ, Calvert SB, et al. A placebo-controlled randomized trial of warfarin in idiopathic pulmonary fibrosis. Am J Respir Crit Care Med 2012; 186: 88-95.

98 Capodanno D, Bhatt DL, Goto S, et al. Safety and efficacy of protease-activated receptor- 1 antagonists in patients with coronary artery disease: a meta-analysis of randomized clinical trials. J Thromb Haemost 2012; 10: 2006-2015.

99 Pusztazeri MP, Seelentag W, Bosman FT. Immunohistochemical expression of endothelial markers CD31, CD34, von Willebrand factor and Fli-1 in normal human tissues. J Histochem Cytochem 2006; 54: 385-395.

100 Yamashita M, Iwama N, Date F, et al. Characterization of lymphangiogenesis in various stages of idiopathic diffuse alveolar damage. Hum Pathol 2009; 40: 542-551.

101 Sozio F, Rossi A, Weber E, et al. Morphomtric analysis of intralobular, interlobular and pleural lymphatics in normal human lung. J Anat 2012; 220: 396-404.

102 Marchetti C, Poggi P, Clement MG, et al. Lymphatic capillaries of the pig lung: TEM and SEM observations. Anat Rec 1994; 238: 368-373.

103 Hainis KD, Sznajder JI, Schraufnagel DE. Lung lymphatics cast from the airspace. Am J Physiol 1994; 267: L199-L205.

104 Souza CA, Muller NL, Lee KS, et al. Idiopathic interstitial pneumonias: prevalence of mediastinal lymph node enlargement in 206 patients. Am J Roentgenol 2006; 186: 995-999.

105 Teles-Grilo ML, Leite-Almeida H, Martins dos Santos J, et al. Differential expression of collagens type I and type IV in lymphangiogenesis during the angiogenic process associated with bleomycin induced pulmonary fibrosis in rat. Lymphology 2005; 38: 130-135. 
106 El-Chemaly S, Malide D, Zudaire E, et al. Abnormal lymphangiogenesis in idiopathic pulmonary fibrosis with insights into cellular and molecular mechanisms. Proc Natl Acad Sci USA 2009; 106: 3958-3963.

107 Ebina M, Shibata N, Ohta H, et al. The disappearance of subpleural and interlobular lymphatics in IPF. Lymphat Res Biol 2010; 8: 199-207.

108 Sueblinvong V, Loi R, Eisenhauer PL, et al. Derivation of lung epithelium from human cord blood-derived mesenchymal stem cells. Am J Respir Crit Care Med 2008; 177: 701-711.
109 Wang D, Haviland DL, Burns AR, et al. A pure population of lung alveolar epithelial type II cells derived from human embryonic stem cells. Proc Natl Acad Sci USA 2007; 104: 4449-4454.

110 Spitalieri P, Quitadamo MC, Orlandi A, et al. Rescue of murine silica-induced lung injury and fibrosis by human embryonic stem cells. Eur Respir J 2012; 39: 446-457.

111 Ortiz LA, Gambelli F, McBride CS. Mesenchymal stem cells engraftment in lung is enhanced in response to bleomycin exposure and ameliorates its fibrotic effects. Proc Natl Acad Sci USA 2003; 100: 8407-8411. 